\title{
Perfil epidemiológico dos pacientes vítimas de trauma atendidos no município de Vitória da Conquista entre os anos de 2017 e 2018
}

\author{
Epidemiological profile of trauma victims treated in the city of Vitória da Conquista \\ between the years 2017 and 2018
}
Perfil epidemiológico de las víctimas de trauma atendidas en la ciudad de Vitória da Conquista entre los años 2017 y 2018

Gabriel Campos Lôbo ${ }^{1 *}$, Cinara Dourado Pereira Correia Santos ${ }^{1}$, Thiago Reis Rocha ${ }^{2}$, Vandressa Lopes da Silva1, Ana Carolina da Silva Soares Martins ${ }^{1}$, Gustavo Oliveira Castro ${ }^{1}$, Isadora Morais

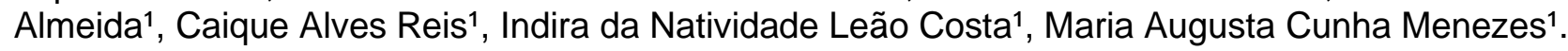

\section{RESUMO}

Objetivo: Este trabalho buscou descrever o perfil epidemiológico das vítimas de trauma atendidas no município de Vitória da Conquista, Bahia, no período de 2017 a 2018. Métodos: Trata-se de um estudo descritivo, quantitativo e retrospectivo, com medidas agregadas calculadas a partir de dados secundários. Para isso, foram utilizadas informações sobre óbitos e internações do Sistema de Informações sobre Mortalidade (SIM/SUS) e do Sistema de Informações Hospitalares (SIH/SUS). Resultados: Percebeu-se que os homens foram os mais acometidos $(80,3 \%)$, em especial os jovens, sendo que aqueles com faxa etária entre 15 - 59 anos corresponderam a $90,4 \%$ de todas as internações dos pacientes do sexo masculino. Além disso, ficou evidente que o traumatismo intracraniano é a etiologia traumática que mais gera custos para o sistema de saúde, constituindo $51 \%$ do valor total. Conclusão: Foi possível perceber que os dados da cidade de Vitória da Conquista foram condizentes com os dados evidenciados na literatura, corroborando com o fato de que a população economicamente ativa, em especial os do sexo masculino, são os mais acometidos por trauma.

Palavras-chave: Epidemiologia, Suporte avançado de vida no trauma, Emergência.

\begin{abstract}
Objective: This work sought to describe the epidemiological profile of trauma victims treated in the city of Vitória da Conquista, Bahia, from 2017 to 2018. Methods: This is a descriptive, quantitative and retrospective study, with aggregate measures calculated from secondary data. For this, information on deaths and hospitalizations from the Mortality Information System (SIM / SUS) and the Hospital Information System (SIH / SUS) were used. Results: It was noticed that men were the most affected (80.3\%), especially young people, with those aged between 15 - 59 years corresponding to $90.4 \%$ of all hospitalizations of male patients. In addition, it was evident that intracranial trauma is the traumatic etiology that most generates costs for the health system, constituting $51 \%$ of the total value. Conclusion: It was possible to perceive that the data from the city of Vitória da Conquista were consistent with the data evidenced in the literature, corroborating with the fact that the economically active population, especially those of the male sex, are the most affected by trauma.
\end{abstract}

Key words: Epidemiology, Advanced trauma life support, Emergencies.

\section{RESUMEN}

Objetivo: Este trabajo buscó describir el perfil epidemiológico de las víctimas de trauma atendidas en la ciudad de Vitória da Conquista, Bahía, de 2017 a 2018. Métodos: Se trata de un estudio descriptivo, cuantitativo y retrospectivo, con medidas agregadas calculadas a partir de datos secundarios. Para ello se utilizó información de defunciones y hospitalizaciones del Sistema de Información de Mortalidad (SIM / SUS)

${ }^{1}$ Faculdades Santo Agostinho, Vitória da Conquista - BA. *E-mail: gabrielclobo17@gmail.com

2 Hospital Geral de Ipiaú (HGI), Ipiaú - BA.

SUBMETIDO EM: 2/2021 
y del Sistema de Información Hospitalaria (SIH / SUS). Resultados: Se observó que los hombres fueron los más afectados $(80,3 \%)$, especialmente los jóvenes, correspondiendo los de 15 a 59 años al $90,4 \%$ del total de hospitalizaciones de pacientes varones. Además, se evidenció que el trauma intracraneal es la etiología traumática que más genera costos para el sistema de salud, constituyendo el $51 \%$ del valor total. Conclusión: Se pudo percibir que los datos de la ciudad de Vitória dan Conquista fueron consistentes con los datos evidenciados en la literatura, corroborando el hecho de que la población económicamente activa, especialmente la del sexo masculino, es la más afectada por el trauma.

Palabras clave: Epidemiología, Atención de apoyo vital avanzado en trauma, Urgencias médicas.

\section{INTRODUÇÃO}

A entidade clínica trauma é caracterizada por modificações na estrutura ou desequilíbrio fisiológico do organismo induzido pela troca de energia entre os tecidos e o meio (BATISTA SE, et al., 2006). Relatos sobre esse tipo de agravo são tão antigos quanto a história do homem, sendo que durante muitos séculos, as guerras foram a principal etiologia para ele, porém, com o avanço tecnológico e o desenvolvimento das sociedades, outras etiologias começaram a se destacar, como por exemplo, o acidente automobilístico (CARVALHO IC e SARAIVA IS, 2015). O óbito ocasionado pelo trauma pode acontecer em momentos distintos e o tratamento precoce é fator decisivo para a sobrevida do paciente, visto que quando realizado de forma eficiente, oferece o suporte necessário para a manutenção da homeostae do organismo.

O primeiro pico de óbito ocorre nos primeiros segundos a minutos em que ocorreu o acidente, sendo caracterizado por lesões extremamente fatais, como ruptura esplênica, hematoma subdural, hematoma epidural, lacerações no baço, lacerações no fígado e hemopneumotórax. Já o segundo, acontece horas após o acidente traumático com lesões que são graves e fatais caso o paciente não receba os devidos cuidados. E o terceiro pico ocorre dias e semanas após o acidente e as principais causas são as falências de orgãos e infecções generalizadas, como a sepse (ALVAREZ BD, 2016). Os ciclistas, os motociclistas e também os pedestres, correspondem aos grupos mais expostos aos acidentes automobilisticos.

Cerca de 270.000 pedestres vão a óbito nas ruas por causa de acidentes de trânsito, sendo representados por $22 \%$ de todas as mortes de trânsito mundial. Em sua maioria, esses acidentes são fatais, mas podem ainda, provocar lesões irreversíveis, sequelas incapacitantes e que podem gerar consequências psiquicas e emocionais as vítimas (PINTO LW, 2016). Alguns comportamentos arriscados podem potencializar o risco aos acidentes de trânsito e provocar traumas irreversíveis às vítimas.

Escolares menores de 18 anos sem a devida autorização para dirigir e com comportamentos irresponsáveis, como manobras ilegais, utilização de drogas, o próprio descuido com o veículo, a forte tendência a autoconfiança e superestimar as próprias habilidades são alguns dos fatores que aumentam a prevalência dos acidentes de trânsito no Brasil e são responsáveis por lesões graves, irreversíveis e até o óbito (MOREIRA MR, 2018). Outros fatores que também podem ser associados com o aumento do risco de acidentes traumáticos no trânsito, são a utilização de celular durante a condução do veículo e a associação de bebidas alcoolicas e a direção. Sendo essa ultima uma das principais causas de acidentes, foi estabelecido leis e aparatos legais que proibem a ingestão de alcool e a direção de veículos de forma concomitante.

A exemplo disso, a lei oํ 11.705/2008, também conhecida como a lei seca regulamenta a tolerância zero para a alcoolemia nos estados brasileiros, tornando inaceitável qualquer quantidade de álcool no organismo, ocasionando multas (REIS PA, 2020). Segundo a Organização Pan-Americana de Saúde (OPAS), cerca de 5,8 milhões de pessoas morrem por trauma no mundo, $32 \%$ a mais que a somatória das mortes de três grandes endemias (malária, tuberculose e a Síndrome da Imunodeficiência Adquirida (AIDS), sendo considerada por muitos autores, como a epidemia do século XXI, acometendo diversos países, independente do desenvolvimento socioeconômico.

Observa-se então, que o trauma é um problema de saúde que possui dimensão internacional, além disso, provoca consequências consideráveis no âmbito social, já que acomete, em sua maioria, os indivíduos economicamente ativos, sendo o agravo à saúde que mais consome anos de vida útil (SILVEIRA ES e 
O'DWYER G, 2017). No Brasil, os traumas provocados por acidentes automobilisticos são considerados a segunda maior causa de morte quando analisados os óbitos por motivos externos. Somente no ano de 2014 foram registrados 43,8 óbitos por acidente e 96.292 internações hospitalares de motociclistas.

Ademais, a maior ocorrência das lesões envolvendo acidentes de trânsito ocorreram em indivíduos com idade entre 15 a 39 anos de idade e isso causou uma despesa em torno de $R \$ 126$ milhões de reais ao Sistema Único de Saúde (MASCARENHAS MDM, 2016). A região centro-oeste ocupa o primeiro lugar com a maior taxa de óbitos por 100.000 habitantes em relação aos acidentes de trânsito. Indiscutivelmente, os homens são os mais atingidos, sendo ainda, a população negra, adultos jovens,motociclistas e individuos com baixa escolaridade os que mais vão a óbito por esse motivo. Ao analisar todas as regiões do Brasil, cerca de um milhão de anos potenciais de vida foram perdidos somente no ano de 2013, especialmente entre os anos de 20 a 29 anos (ANDRADE SS e MELLO-JORGE MH, 2016).

Alvarez BD, et al. (2016) registraram que de uma amostra com 200 pacientes vítimas de trauma atendidos nos serviços de urgência e emergencia $73,5 \%$ são do sexo masculino, compondo a população economicamente ativa com idade entre 15 a 40 anos. Os autores afirmam ainda que principais etiologias são a violência e o acidente autociclistico, mas outras etiologias podem ser levadas em consideração, como o acidente automobilístico, atropelamentos, queda de altura, agressão física, queda da própria altura e acidentes de bicicleta (ALVAREZ BD, et al., 2016). O aumento dos gastos relacionados a emergências traumáticas, reabilitação e assistência são signiticativamente maiores quando comparados com os gastos relacionados a maioria dos procedimentos médicos convencionais.

Na região no nordeste brasileiro, por exemplo, os custos de internações devido a acidentes de trânsito são $89 \%$ maiores que os custos relacionados a internações por outros motivos. Ao relacionar esta comparação a nível nacional, a diferença entre as internações traumáticas e outros procedimentos chegam a $37 \%$ (CASTRO RRM, 2013). É importante salientar ainda que o impacto social é o fato de, com muita frequência, o evento traumático ter como consequências, além do óbito, as sequelas incapacitantes e permanentes, o que propicia um aumento nos gastos públicos não só com as medidas terapêuticas, mas também de reabilitação, além da diminuição do período de atividade laboral dos pacientes.

Esses impactos economicos afetam de forma significativa a economia nacional não somente de forma direta, mas indiretamente também, especialmente quando relacionados as oportunidades perdidas.Ademais, há ainda gastos com reparos materiais, custos de ações jurídicas, tratamentos, serviços hospitalares, custos médicos e custos ocasionados pelo óbito (TISCHER V, 2019). Credo PF e Félix JV (2012), em estudo, afirmaram que de um total de 801 prontuários avaliados e que foram vítimas de graves traumas, 624 eram do sexo masculino e que a maior incidência das lesões compreendia a faixa etária entre 15 a 44 anos de idade. Além disso, quando analisados os traumas em idades superiores a 75 anos, a maior prevalência foi do sexo feminino.

Os autores discutiram que os principais mecanismos do trauma foram representados por acidentes de trânsito, quedas e também a violência (CREDO PF e FÉLIX JV, 2012). Identificar o perfil dos pacientes atendidos é de fundamental importância para melhorar a assistência e direcionar as ações das políticas públicas em saúde. Dessa forma, objetivou-se nesse estudo, traçar o perfil epidemiológico dos pacientes vítimas de trauma atendidos no município de Vitória da Conquista - Bahia, nos anos de 2017 e 2018, identificando suas variáveis sociais, demográficas, clínicas e financeiras, bem como correlacionar os dados encontrados com dados descritos na literatura.

\section{MÉTODOS}

O presente constructo trata-se de um estudo descritivo, quantitativo e retrospectivo, com medidas agregadas calculadas a partir de dados secundários. Para isso, foram utilizadas informações sobre óbitos e internações do Sistema de Informações sobre Mortalidade (SIM/SUS) e do Sistema de Informações Hospitalares (SIH/SUS), ambos disponíveis na base de dados do Departamento de Informática do Sistema Único de Saúde (Datasus), plataforma do Ministério da Saúde. 
Em todo território brasileiro, os registros de informação em saúde, bem como o impacto dos acidentes traumáticos são disponibilizados nos sistemas de informação do Ministério da Saúde. Dados como taxas de mortalidade, hospitalizações, acidentes de trânsito são alimentados e disponibilizados virtualmente em plataformas acessíveis a toda a população, que podem ser acessados a qualquer momento (RIOS PAA, et al., 2019). Os dados utilizados para essa pesquisa correspondem as internações hospitalares, óbitos, idade dos pacientes traumáticos, sexo, cor da pele, taxa de mortalidade, média de dias internado, valor médio por internamento e valor total gasto por etiologia do trauma, no período de 2017 a 2018, no município de Vitória da Conquista - Bahia.

Os traumas considerados no estudo, com seus respectivos CID's, foram: traumas de regiões não específicas do corpo (T009), traumatismo intracraniano (S06), lesões por esmagamentos e amputações traumáticos (T147) e traumatismos de outros órgãos internos (S368). Esses CID's foram utilizados pois eram os que apresentavam a melhor população amostral e estão dentro do universo estudados nesse trabalho. Outros tipos de trauma como agressões, quedas e acidente de transporte não foram utilizados no estudo, pois não haviam dados na plataforma do Datasus, sendo uma importante limitação do estudo.

Por se tratar de um trabalho realizado com dados secundários, não se teve acesso ao nome dos pacientes, o que garantiu o anonimato dos mesmos. Além disso, os dados utilizados estão disponíveis na internet e são de domínio público, por isso não foi necessária a submissão do trabalho ao Comitê de Ética em pesquisa.

\section{RESULTADOS}

As características do perfil social e demográfico, como a maior prevalência do sexo masculino e de ser uma amostra constituída, em sua maioria, pela população economicamente ativa com idade entre 15-59 anos de idade (Tabela 1). No presente estudo, os dados coletados no Datasus correspondem a um total de quatro etiologias (Tabela 2).

Além disso, foi constatado que a média de dias de internação foi variável, segundo a causa do trauma, sendo: 5,0 dias para os pacientes vítimas de traumas não específicos do corpo, 8,4 dias para os pacientes vítimas de traumatismo intracraniano, 2,3 dias para lesões por esmagamento ou amputação traumática e de 7,1 para os pacientes internados por trauma de outros órgãos internos.

Tabela 1 - Análise descritiva do perfil sociodemográfico dos pacientes vítimas de trauma atendidos em Vitória da Conquista- BA, 2017 a 2018.

\begin{tabular}{ccc}
\hline Variáveis & $\mathbf{n}$ & $\%$ \\
\hline Sexo & & \\
\hline Feminino & 414 & 19,6 \\
Masculino & 1696 & 80,3 \\
\hline Cor & & \\
\hline Branco & 29 & 1,38 \\
Preto & 2 & 0,09 \\
Pardo & 269 & 12,8 \\
Amarelo & 3 & 0,14 \\
Sem informações & 1791 & 85,5 \\
\hline Idade & & \\
\hline$<1-14$ & 204 & 9,6 \\
$15-59$ & 1534 & 72,7 \\
$60->80$ & 372 & 17,6
\end{tabular}

Fonte: LÔBO GC, et al., 2021; dados extraídos do Sistema de Informações sobre Mortalidade (SIM/SUS) e Sistema de Informações Hospitalares (SIH/SUS). 
Na Tabela 2 está evidenciada a importância que cada etiologia citada possui, tanto em números totais de internamento, quanto com relação ao prognóstico desses pacientes, analisando o número de óbitos. Vale ressaltar que o valor gasto com os pacientes vítimas de trauma é de grande significância, sendo que a plataforma do Ministério da Saúde informa que nesses dois anos (2017 - 2018), o valor total gasto, considerando as quatro situações clínicas apresentadas foi de 2.993.987,25 reais, sendo que o valor gasto com o traumatismo cranio encefálico representou $51 \%$ desse valor (Tabela 3 ).

Ainda, é possível demonstrar uma comparação entre a quantidade de internamentos no município de Vitória da Conquista e o total no estado da Bahia. Sendo possível, assim, revelar o impacto dos pacientes atendidos nesse município dentro do cenário estadual, pois Vitória da Conquista correspondeu a 4,7\% de todos os internamentos, nos anos de 2017 e 2018, merecendo destaque, o traumatismo cranioencefálico, que representou $6 \%$ de todos os traumatismos cranioencefálicos do Estado (Gráfico 1).

Tabela 2 - Análise descritiva das diferentes etiologias traumáticas e óbitos registrados no município de Vitória da Conquista-BA, de 2017 a 2018.

\begin{tabular}{ccc}
\hline Variáveis & $\mathbf{n}$ & $\%$ \\
\hline Etiologia do trauma & & 39,1 \\
Outros traumas de regiões não específicas & 826 & 36,8 \\
Traumatismo intracraniano & 778 & 9,57 \\
Lesões por esmagamento e amputação traumática & 202 & 14,4 \\
Traumatismo de outros órgãos internos & 304 & \\
\hline Óbitos por etiologia & & 19,1 \\
\hline Outros traumas de regiões não específicas & 26 & 61 \\
Traumatismo intracraniano & 83 & 1,4 \\
Lesões por esmagamento e amputação traumática & 2 & 18,3 \\
Traumatismo de outros órgãos internos & 25 &
\end{tabular}

Fonte: LÔBO GC, et al., 2021; dados extraídos do Sistema de Informações sobre Mortalidade (SIM/SUS) e Sistema de Informações Hospitalares (SIH/SUS).

A Tabela 1 demonstra que a população mais acometida é a economicamente ativa, em especial, do sexo masculino. Outrossim, dados colhidos mostram que dos pacientes vítimas de trauma, os homens são os que representam uma maior quantidade de óbitos: do total de 136 óbitos, 80,8\% são do sexo masculino e a causa que possui uma maior taxa de letalidade é o traumatismo intracraniano, correspondendo a cerca de $61 \%$ (Tabela 3).

Tabela 3 - Custo do paciente vítima de trauma para o SUS, segundo etiologia no município de Vitória da Conquista-BA, 2017 a 2018.

\begin{tabular}{ccc}
\hline Variáveis & Valor (R\$) & $\%$ \\
\hline Etiologia & & 20 \\
\hline Outros traumas de regiões não específicas & $612.074,26$ & 51 \\
\hline Traumatismo intracraniano & $1.539 .136,33$ & 5 \\
\hline Lesões por esmagamento e amputação traumática & $136.409,82$ & 24 \\
\hline Traumatismo de outros órgãos internos & $706.366,84$ & $\mathbf{1 0 0}$ \\
\hline Total & $\mathbf{2 . 9 9 3 . 9 8 7 , 2 5}$ &
\end{tabular}

Fonte: LÔBO GC, et al., 2021; dados extraídos do Sistema de Informações sobre Mortalidade (SIM/SUS) e Sistema de Informações Hospitalares (SIH/SUS). 
No Gráfico 1 é demonstrada a comparação entre o total de vítimas por internações devido ao trauma, tanto no município de Vitória da Conquista quanto no estado da Bahia durante os anos de 2017 e 2018 . O traumatismo intracraniano foi a segunda principal causa de internações hospitalares nas duas regiões, remetendo a significativa importância para os setores de saúde. A principal causa estabelecida das internações foram os traumas em regiões não especificadas, porém outras causas também foram registradas, tais como amputações traumáticas, lesões esmagadoras e traumas em outros órgãos.

Gráfico 1 - Comparação entre o número de internações por trauma no estado da Bahia e em Vitória da Conquista - BA, de acordo com as etiologias de 2017 a 2018.

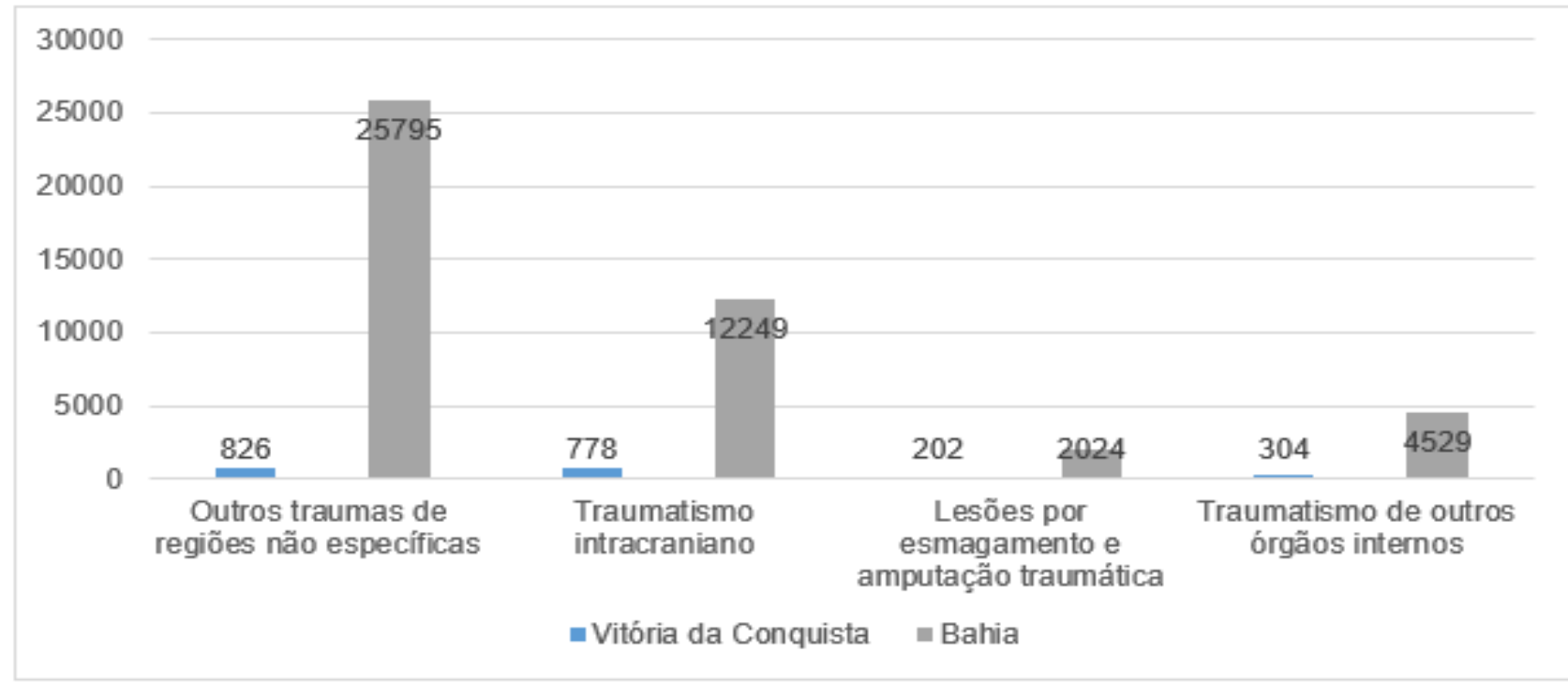

Fonte: LÔBO GC, et al., 2021; dados extraídos do Sistema de Informações sobre Mortalidade (SIM/SUS) e Sistema de Informações Hospitalares (SIH/SUS).

\section{DISCUSSÃO}

Considerando o exposto, observa-se que a história do trauma é um reflexo direto da história da humanidade, sendo considerado como a epidemia do século XXI. Atualmente, aproximadamente 60 milhões de pessoas são acometidas por algum tipo de trauma, estando entre as principais causas a violência e 0 acidente de trânsito. Os dados encontrados na literatura expressam, de forma unânime, a realidade nacional e internacional do perfil dos pacientes vítimas de trauma, sendo constituído, em sua maioria, por jovens do sexo masculino, com uma faixa etária entre 5 e 40 anos, e composta, majoritariamente, por indivíduos da parcela populacional economicamente ativa (PRAÇA WR, 2015).

A análise da amostra coletada, evidenciou ainda que a população dos pacientes vítimas de trauma atendidos no município de Vitória da Conquista é, majoritariamente, de jovens do sexo masculino, em todas as etiologias traumáticas analisadas tanto nas internações como no número de óbitos. Tal realidade é condizente com os dados apresentados na literatura, onde os homens jovens são os mais acometidos e os que mais possuem um desfecho letal, como descrito por Padovani em seu estudo, no qual quase $82 \%$ da amostra era do sexo masculino (PADOVANI C, et al., 2014).

Oliveira AL, et al. (2015) reiteram esses dados. Os autores avaliaram 100 pacientes vítimas de traumas e registraram um total de 91 indivíduos do sexo masculino com idade superior a 20 anos. A população de intrevistados conduziam motocicletas e automóveis no momento do acidente e referem que a imprudência foi o principal motivo que os levou ao acidente.

Os motociclistas são as principais vítimas de trauma, sendo representados por $44,2 \%$ dos feridos no ano de 2011 na cidade de Belo Horizonte (OLIVEIRA AL, et al., 2015). Ao destacar o traumatismo intracraniano, uma das etiologias avaliadas nesse estudo, observa-se a alta relação existente entre esse agravo e o óbito, 
revelando uma alta taxa de mortalidade (CONSTÂNCIO JF, et al., 2018). No presente estudo, o traumatismo cranioencefálico correspondeu a cerca de $37 \%$ dos internamentos de pacientes vítimas de trauma, levando a aproximadamente $61 \%$ de todos os óbitos. Percebe-se na literatura que alguns autores tentam explicar a relação entre o numero significativo de jovens do sexo masculino que são vítimas de trauma.

As teorias que fundamentam esse acontecimento são: inexperiência, busca de emoções, prazer pessoal, experimentar sensações de risco, além do uso e abuso de álcool e outras drogas, ou seja, justificada no padrão de comportamento social e cultural que está relacionado ao sexo e à idade (CANOVA JCM, et al., 2010).

É perceptível que o trauma constitui um dos maiores desafios para a saúde pública, pois acarreta consequências para o individuo traumatizado, tanto social quanto economicamente e também para a sociedade e o sistema de saúde. Pois, além das lesões, o trauma pode ocasionar óbitos e incapacidade física, seja ela permanente ou temporária, além de possuir um alto custo com a recuperação e reabilitação do doente (SANTOS LFS, et al., 2016).

Esse estudo evidenciou que os diferentes tipos de trauma são resposáveis por desencadear diferentes impactos sociais, desde o valor gasto por internação do paciente até a diminuição da vida útil, bem como do tempo de contribuição econômica, ou seja, perda de anos de vida produtiva. Dessa forma, nota-se que os custos totais relacionados ao trauma, excedem os gastos relacionados ao câncer e também os gastos relacionados as doenças cardiovasculares (TOLOTTI VC e SILVA LAA, 2004; CHALPYA PL, et al., 2011).

Diante do exposto, merece destaque o traumatismo cranioencefálico, responsável por cerca de $51 \%$ do total de gastos com o paciente vítima de trauma atendido em Vitória da Conquista. Corroborando com o que foi dito acima, pesquisas revelam que o traumatismo cranioencefálico é a principal causa de morte e sequelas na faixa etária de 1 a 44 anos de idade (CONSTÂNCIO JF, et al., 2018).

Assim, o trauma é uma importante causa de internamento e pode ser responsável por cerca de $40 \%$ de todos os leitos de internação disponibilizados, a depender do serviço (MELO JRT, et al., 2004). Por fim, vale destacar a importância da criação do Serviço de Atendimento Móvel de Urgência e Emergência (SAMU), que foi implantado em Vitória da Conquista no ano de 2004, pois é evidente que o tempo decorrido entre o evento traumático e o atendimento pré-hospitalar, bem como o tempo necessário para o transporte desse paciente para o tratamento hospitalar adequado é de extrema importância no que diz respeito a sobrevida da vítima de trauma.

Observa-se que esse atendimento prestato é fator decisivo para reduzir, não só a mortalidade, mas também a ocorrência de sequelas aos pacientes vítimas do trauma (CARVALHO IC e SARAIVA IS, 2015). Vale destacar alguns protocolos que são efetivos em reduzir os desfechos ruins nos pacientes vítimas de trauma, como o Atendimento Pré-Hospitalar ao Traumatizado (PHTLS) e o Suporte Avançado de Vida no Trauma (ATLS) (ALTO LSM, 2007).

Alto LSM (2007) discutiu que, após a implantação do ATLS o número geral de óbitos reduziu cerca de $17 \%$, sendo que a maior redução foi nas mortes após a primeira hora do trauma, mas em contrapartida, houve um aumento importante na quantidade de pacientes que foram ao óbito após 24 horas (ALTO LSM, 2007). Ademais, observa-se o impacto que esse agravo gera ao Sistema Único de Saúde, assim como em outras esferas do governo, devido ao acometimento, muitas vezes com sequelas permanentes da população economicamente ativa.

Vale lembrar que esse estudo possuiu uma importante limitação decorrente da metodologia utilizada: a subnotificação da plataforma do Datasus, sendo necessário um estudo com maior acurácia para a determinação do perfil de pacientes atendidos na cidade em questão. Destaca-se, dessa forma, a importância do preenchimento adequado do prontuário, bem como o lançamento dos dados no sistema. Ademais, observa-se o impacto que esse agravo gera no Sistema Único de Saúde, assim como em outras esferas do governo, devido ao acometimento, muitas vezes com sequelas permanentes da população economicamente ativa. 


\section{CONCLUSÃO}

Os resultados desse estudo permitiram descrever o perfil clínico-epidemiológico do paciente vítima de trauma no município de Vitória da Conquista-BA durante o período de 2017 e 2018. Assim, observa-se que o perfil geral das vítimas na população analisada corresponde ao perfil nacional, sendo constituída, majoritariamente, por pacientes adultos jovens e do sexo masculino, bem como o traumatismo cranioencefálico como maior representante percentual de todos os óbitos avaliados. Desta forma, baseandose nessas constatações, destaca-se que o trauma possui um impacto significativo na saúde pública. Por isso, é importante que medidas sejam adotadas para prevení-lo, como campanhas educativas e ações de fiscalização efetivas.

\section{REFERÊNCIAS}

1. ALBINO R, RIGGENBACH V. Atendimento hospitalar inicial ao politraumatizado. Arquivos catarinenses de medicina, 2004; 33(3): 18-22.

2. ALTO LSM. Análise do impacto do programa ATLS (Advanced Trauma Life Support) no atendimento do traumatizado em cidade de pequeno porte no Brasil, SP. Tese (doutorado em emergências clínicas) - Faculdade de medicinaUniversidade de São Paulo, São Paulo, 2008.

3. ALVAREZ BDA, et al. Análise do Revised Trauma Score (RTS) em 200 vítimas de diferentes mecanismos de trauma. Revista do colégio brasileiro de cirurgiões, 2016; 43(5): 334-340.

4. ANDRADE SSCA, MELLO-JORGE MHP. Mortalidade e anos potenciais de vida perdidos por acidentes de trânsito no Brasil. Revista de saúde pública, 2016; 50 (59): 1-9.

5. AZEVEDO ALCS. Gerenciamento do cuidado de enfermagem em unidade traumática, SP. Dissertação (mestrado em enfermagem fundamental) - Escola de enfermagem de Ribeirão Preto, Ribeirão Preto, 2010.

6. BATISTA SEA et al. Análise comparativa entre os mecanismos de trauma, as lesões e o perfil de gravidade das vítimas, em Catanduva-SP. Revista do colégio brasileiro de cirurgiões, 2006; 33(1):6-10.

7. CANOVA JCM, et al. Traumatismo cranioencefálico de pacientes vítimas de acidentes de motocicletas. Arquivo ciência e saúde, 2010; 17(1): 9-14.

8. CONSTÂNCIO JF, et al. Perfil clínico-epidemiológico de indivíduos com histórico de traumatismo cranioencefálico. Revista baiana de enfermagem, 2018; 32: 1-10.

9. CARVALHO ICCM, SARAIVA IS. Perfil das vítimas de trauma atendidas pelo serviço de atendimento móvel de urgência. Revista interdisciplinar, 2015; 8(1): 137-148.

10. CASTRO RRM, et al. Perfil dos pacientes na enfermaria de ortopedia de um hospital público de Salvador-Bahia. Acta ortopédica brasileira, 2013; 21(4): 191-194.

11. COSTA-VAL R, et al. Reflexões sobre o trauma cardiovascular civil a partir de um estudo prospectivo de 1000 casos atendidos em um centro de trauma de nível I. Revista do colégio brasileiro de cirurgiões, 2008; 35(3):162-167.

12. CREDO PFD, FELIX JVC. Perfil dos pacientes atendidos em um hospital de referência ao trauma em Curitiba: implicações para a enfermagem. Cogitare enfermagem, 2012; 17(1): 127-131.

13. CHALYA PL, et al. Trauma admissions to the intensive care unit at a reference hospital in Northwestern Tanzania. Scandinavian journal of trauma, 2011; 19(61): 1-7.

14. LAURENTI R, et al. A classificação internacional de doenças, a família de classificações internacionais, a CID-11 e a síndrome pós-poliomielite. Arquivos de neuro-psiquiatria, 2013; 71(9): 3-10.

15. MASCARENHAS MDM et al. Características de motociclistas envolvidos em acidentes de transporte atendidos em serviços públicos de urgência e emergência. Ciência e saúde coletiva, 2016; 21(12): 3661- 3671.

16. MELO JRT, et al. Caracteristicas dos pacientes com trauma cranioencefálico na cidade de Salvador, Bahia, Brasil. Arquivos de neuropsiquiatria, 2004; 62(3): 711-715.

17. MOREIRA MR, et al. Mortalidade por acidentes de trânsito em adolescentes e jovens, Brasil, 1996-2015: chegaremos ao ODS 3.6?. Revista ciência e saúde, 2018; 23(9): 2785-2796.

18. NATHENS AB et al. Development of trauma systems and effect on outcomes after injury. Lancet, 2004; 363(9423): 1794-1801.

19. OLIVEIRA AL, et al. Caracteristicas dos motociclistas envolvidos em acidentes entre motocicleta e automóvel. Revista da associação médica brasileira, 2015; 61(1): 61-64.

20. ORGANIZAÇÃO MUNDIAL DA SAÚDE, ORGANIZAÇÃO PANAMERICANA DE SAÚDE. Classificação estatística internacional de doenças e problemas relacionados a saúde: CID-10, décima revisão. Trad de Centro Colaborador da OMS para a Classificação de Doenças em Português. Vol 2, 3ed. São Paulo: EDUSP, 1996.

21. PADOVANI C, et al. Perfil dos pacientes politraumatizados graves atendidos em um serviço público de referência. Arquivo de Ciências da Saúde, 2014; 21(3): 41-45.

22. PAIVA JÁ, et al. Citocinas e moléculas de adesão na avaliação de politraumatizados graves. Revista portuguesa de ortopedia e traumatologia, 2013; 21 (2): 121-145.

23. PANICHI RMD, WAGNER A. Comportamento de risco no trânsito: revisando a literatura sobre as variáveis preditoras da condução perigosa na população juvenil. Interamerican journal of psychology, 2006; 40(2): 159-166.

24. PRAÇA WR. Vítimas de trauma no DF: Perfil epidemiológico e atendimento pré e intra-hospitalar pelo SAMU, Brasília. Trabalho de conclusão de curso (graduação em enfermagem) - Universidade de Brasilia, Brasília, 2015. 
25. PRAÇA WR, et al. Perfil epidemiológico e clínico de vítimas de trauma em um hospital do Distrito Federal, Brasil. Revista prevenção de infecção e saúde, 2017; 3(1): 1-7.

26. RIOS PAA, et al. Fatores associados a acidentes de trânsito entre condutores de veículos: achados de um estudo de base populacional. Ciencia e saúde coletiva, 2020; 25(3): 943-955.

27. RIOS PAA, et al. Acidentes de trânsito entre motoristas: incidência e diferenças entre motociclistas e motoristas em estudo de base populacional. Revista brasileira de epidemiologia, 2019; 22: 1-13.

28. PINTO LW, et al. Atendimento de urgência e emergência a pedestres lesionados no trânsito brasileiro. Ciência e saúde coletiva, 2016; 21(12): 3673-3682.

29. SANTOS LFS, et al. Estudo epidemiológico do trauma ortopédico em um serviço público de emergência. Caderno saúde coletiva, 2016; 24(4): 397-403.

30. SANTOS WJ, et al. Alcohol and risky behavior in traffic among motorcyclists involved in accidents in a city in northeastern Brazil. Traffic injury prevention, 2019; 20(3): 233-237.

31. SENEFONTE FR, et al. Amputação primária no trauma: perfil de um hospital da região centro-oeste do Brasil. Jornal vascular brasileiro, 2012; 11(4): 269- 276.

32. SILVA AMA, SHAMA SFMS. Epidemiologia do trauma em atendimentos do SAMU, Novo Hamburgo- RS no primeiro trimestre de 2015. Revista saúde e pesquisa, 2017; 10(3): 539-548.

33. SILVEIRA ES, O'DWYER G. Centro de trauma: modelo alternativo de atendimento às causas externas no estado do Rio de Janeiro. Saúde debate, 2017; 41(112): 243-254.

34. TISCHER V. O custo social e econômico dos acidentes de trânsito com pedestres e ciclistas: estudo de caso do estado de Santa Catarina, Brasil, 2019; 11: 1-14.

35. TOLOTTI VC, SILVA LAA. Caracterização das vítimas de trauma atendidas em emergência hospitalar no norte do estado do Rio Grande do Sul. Revista contexto e saúde, 2004; 4(7): 191-198. 